\title{
Editorial
}

Mycosphere

\section{Editorial - New taxa of wood-inhabiting fungi from the tropics}

\section{Yu-Cheng Dai ${ }^{1}$}

\author{
${ }^{1}$ Institute of Microbiology, PO Box 61, Beijing Forestry University, Beijing 100083, China
}

Dai YC 2017 -New taxa of wood-inhabiting fungi from tropics. Mycosphere 8(6), 560, Doi 10.5943/mycosphere/8/6/1

Wood-inhabiting fungi are not taxonomically a natural taxon, but have a similar ecology (growing on wood) in nature; most of these fungi can decompose cellulose, hemicellulose and lignin in woody plants, and therefore play a key role in nutrient recycling in most forest ecosystems. The major taxa of this group fungi are traditionally known as aphyllophoroid fungi, and share the tough basidiocarps not fleshy as in the Agaricales, but with variable of hymenophore as smooth, poroid, irpicoid, reticulate, merulioid, grandinoid, ondontioid, hydnoid, tuberculate, colliculose and epitheloid etc. In addition, wood-inhabiting fungi have different shapes of fruiting body such as resupinate, effused-reflexed, pileate and stipitate etc. Molecular evidence has shown wood-inhabiting fungi are distinctly polyphyletic; based on the modern taxonomy they belong to orders of Agaricales, Amylocorticiales, Atheliales, Boletales, Auriculariales, Cantharellales, Corticiales, Gloeophyllales, Hymenochaetales, Jaapiales, Polyporales, Russulales, Thelephorales and Trechisporales, but most taxa in Polyporales, Hymenochaetales, Thelephorales, Russulales and Corticiales.

Some members of wood-inhabiting fungi are the serious forest pathogens, e.g. the species of genus Heterobasidion Bref. cause the most destructive forest disease in north hemisphere, and some wood-inhabiting fungi have been used as medicinal herbs, e.g. the selling for various productions of Ganoderma lingzhi Sheng H. Wu, Y. Cao \& Y.C. Dai is over 2 billion US\$ by 2015. Species diversity of wood-inhabiting fungi in boreal and temperate areas is relatively well known, however, it is still poorly known in tropics because of less exploration and rich of woody plants. Most wood-inhabiting fungi were reported to grow on an extensive host range, but recent studies showed species in many genera were considered to be host-specific. Recently a greater number of novel genera and species of wood-inhabiting fungi were described from tropics by the application of molecular techniques, and these studies are mostly connected with phylogenies. New taxa are redefined based on a combination of morphology, ecology, host-specific and DNA sequence analysis. Phylogeny on wood-inhabiting fungi in tropics is more important than in boreal and temperate areas, especially for the host-specific groups, because the host tree species in boreal and temperate areas are usually recognized at generic or species level by mycologists, but they are very difficult in tropics.

This issue of Mycosphere includes papers dealing with new taxa of wood-inhabiting fungi found from tropical Asia, Central America and South America, and phylogenetic analyses on these new taxa are included. Ecological discussions on substrate specificity of some genera are provided. 